\title{
La diaspora kazakhe et la politique de rapatriement de la République du Kazakhstan
}

Kazakh Diaspora and Repatriation Politics of the Kazakhstan Republic

La diáspora kazaka y la política de repatriación de la República de Kazajstán

\section{Gulnara Mendikulova}

\section{OpenEdition}

Édition électronique

URL : https://journals.openedition.org/remi/5230

DOI : $10.4000 /$ remi.5230

ISSN : $1777-5418$

Éditeur

Université de Poitiers

Édition imprimée

Date de publication : 1 décembre 2010

Pagination : 153-167

ISBN : 978-2-911627-56-9

ISSN : 0765-0752

\section{Référence électronique}

Gulnara Mendikulova, "La diaspora kazakhe et la politique de rapatriement de la République du

Kazakhstan », Revue européenne des migrations internationales [En ligne], vol. 26 - n³ | 2010, mis en ligne le 01 décembre 2013, consulté le 15 avril 2022. URL : http://journals.openedition.org/remi/5230 DOI : https://doi.org/10.4000/remi.5230 


\section{Note de recherche}

\section{La diaspora kazakhe et la politique de rapatriement de la République du Kazakhstan}

\section{Gulnara MENDIKULOVA ${ }^{1}$}

ujourd'hui les processus inter et intra-ethniques, dus à d'importants bouleversements mondiaux socio-économiques, politiques et culturels, ne cessent de s'intensifier et ils n'épargnent pas le Kazakhstan. Ce pays indépendant et multiethnique apparaît sur la scène internationale comme un État moderne et démocratique grâce à la consolidation et à l'unification de la nation, incluant les populations kazakhes qui vivent à l'extérieur des frontières nationales. Près de 4,5 millions de Kazakhs vivent disséminés à l'étranger, dans les quatorze autres États issus de l'éclatement de l'URSS et vingtcinq autres pays du monde ; 800000 d'entre eux constituent la diaspora et 3,7 millions constituent l'irredenta, c'est-à-dire les Kazakhs qui vivent hors des frontières dans des espaces limitrophes du Kazakhstan (principalement en Fédération de Russie, Chine et Ouzbékistan) depuis des temps plus ou moins anciens, du fait d'ambitions impériales et/ ou de jeux de pouvoir de la part du régime tsariste puis du pouvoir soviétique.

Irredenta et diaspora partagent un trait commun : chacune considère le Kazakhstan actuel comme sa patrie historique dont elle estime faire partie en qualité de ressortissant de droit. Les origines de la diaspora appartiennent à l'histoire, mais le présent s'inscrit dans le développement du Kazakhstan contemporain.

Cet article est d'autant plus d'actualité que le Kazakhstan, membre de plein droit de la communauté internationale, doit répondre à l'épineuse question des relations, tant au niveau international qu'intergouvernemental, entre trois composantes essentielles : la République du Kazakhstan, les représentants de la diaspora et de l'irredenta, et le pays d'accueil de ces dernières. Diaspora et irredenta sont des éléments très importants de la politique extérieure du Kazakhstan vis-à-vis des pays d'accueil. En tant que minorité ethnique en milieu étranger, la situation des Kazakhs pèse donc dans les relations entre États.

1 Prof. Dr., Director, Centre of Diaspora Studies, The World Association of the Kazakhs, U1. Valikhanova 117, kv. 35, 050010 Kazakhstan Almaty ; gmendikulova@hotmail.com L'article a été traduit du russe au français par Vanessa Balci. 
Les statuts que possèdent les Kazakhs dépendent de la politique d'accueil des gouvernements en place et conditionnent partiellement l'action du Kazakhstan à leur égard sur la scène internationale. Forts d'une expérience d'adaptation et parfois de survie en milieu étranger, dans un contexte nouveau d'une économie de marché, les membres de la diaspora participent au développement socio-économique de leur pays d'origine. Porteurs de savoirs traditionnels, ils sont en mesure de contribuer au renouveau culturel d'une nation kazakhe qui a connu une forte acculturation sous la période soviétique.

Nous abordons ici l'analyse des aspects théoriques et pratiques liés à la diaspora et à l'irredenta kazakhes ainsi que la politique de rapatriement du Kazakhstan.

\section{DIASPORA ET IRREDENTA KAZAKHES}

Sheffer définit la diaspora comme un « groupe appartenant à une minorité ethnique, ayant quitté sa patrie, vivant et agissant dans un pays d'accueil, tout en conservant des liens sentimentaux et matériels forts avec la patrie d'origine » (1986: 3). La diaspora kazakhe résulte de migrations sur de longues distances. D’abord temporaires, elles sont devenues définitives et passent par plusieurs frontières interétatiques : celles du Kazakhstan soviétique, de la Chine, des autres États d'Asie centrale, de l'Afghanistan et de l'Iran, et de là vers le reste du monde. À partir des années 1960, les représentants de la diaspora participent d'un premier exode rural et en quittant les campagnes pour les villes, ils changent d'orientation socio-économique et professionnelle.

Tout au long de son histoire, la diaspora fut, pour des raisons politiques et religieuses, le produit de migrations forcées et violentes et ce, jusque dans les années 1960 où commence à se développer une émigration économique vers l'Europe occidentale, l'Amérique et le golfe Arabo-persique. Jusqu'au début des années 1960, les migrations des Kazakhs se distinguaient par leur caractère collectif, aujourd'hui il s'agit davantage de déplacements individuels, suivis d'un regroupement familial dans le pays d'accueil. Selon Ashkenasi (1990), la diaspora se caractérise par des relations entre trois composantes :

- un groupe ethnique, doté de formes linguistiques, religieuses et historiques propres ;

- une patrie, c'est-à-dire un espace géographique pour lequel le groupe ethnique a un attachement ;

- le pays d'accueil, c'est-à-dire l'État où vit actuellement la diaspora.

Dans le cas de la diaspora kazakhe, on constate que ses membres représentent toutes les catégories sociales de la nation, partagent une même patrie historique, sont de confession musulmane pour la plupart et appartiennent à la famille des peuples turcophones.

Cette diaspora compte environ 800000 individus dispersés dans divers pays d'accueil. Ils ne constituent pas un groupe ethnique vivant dans un espace propre, sauf en Turquie, en Iran ou en Afghanistan où les Kazakhs ont tendance à se regrouper encouragés en cela par les politiques du pays de résidence. Considérés comme une minorité ethnique, ils n'ont jamais pesé sur les politiques locales. 
Parmi les raisons politiques qui ont entraîné les premières dispersions on peut citer les guerres kazakho-djoungares du XVIIIe siècle, les insurrections et les guerres contre l'autocratie tsariste aux XVIIIe et XIXe siècles, le mouvement de libération nationale en Asie centrale contre l'empire tsariste en 1916, l'établissement du pouvoir soviétique et la Guerre civile, le génocide des Kazakhs sous la collectivisation, la lutte contre les communistes chinois au Turkestan oriental (actuel Xinjiang) ou les effets de la Seconde Guerre mondiale. Les raisons économiques qui ont poussé à la migration tiennent à la politique agraire de Stolypine à l'époque tsariste, la destruction du mode de vie nomade traditionnel après l'intégration du Kazakhstan à la Russie et sous la collectivisation soviétique (1928-1932), l'émigration économique des descendants des réfugiés vers les pays d'Europe occidentale et d'Amérique dans les années 1960-1990, l'instabilité économique du Kazakhstan après l'effondrement du système socialiste et de l'économie de l'URSS au début des années 1990 et la transition vers l'économie de marché. Les raisons religieuses sont liées, aussi bien sous les Tsars que sous les Soviets, à l'interdiction pour les fidèles de quitter le pays et d'accomplir le pèlerinage à La Mecque et à Médine, lieux sacrés de l'Islam. Ceux qui arrivaient à partir en pèlerinage tentaient de rester dans les pays d'Orient pour se conformer aux prescriptions de l'Islam. Aujourd'hui encore, dans les villages de Şekerköy et Akhunköy en Turquie, vivent les descendants d'une trentaine de familles kazakhes qui ont accompli le hadj au XIXe siècle et sont restés sur place depuis cette époque (Mendikulova, $2006: 24$ ).

La diaspora est à la fois objet de politique intérieure et sujet des relations internationales. Ses caractéristiques correspondent aux orientations de la politique ethnique qui prend en compte les « co-ethniques » séparés de la nation (l'irredenta), l'ethnicité comme ressource stratégique, les nations sans État, les communautés ethniques transnationales et l'internationalisation des droits des minorités. À ce titre elle est d'une part un élément essentiel du pluralisme ethnique qui joue sur les relations internationales et elle exerce d'autre part une influence sur les conflits ethniques internes (Sheffer, 1986). Ainsi, Milton J. Esman (1990 : 83-88) considère que le premier levier pour un pays en difficulté est d'appeler à l'aide sa diaspora² .

Le second levier s'appuie sur des actions qui ont une influence directe sur les évènements dans le pays d'origine. Elles peuvent relever d'initiatives indépendantes ou être inspirées par le pays d'accueil ou le pays d'origine et contribuer aussi à freiner les intérêts du pays d'accueil comme ceux du pays d'origine. En général, le gouvernement du pays d'origine est plutôt favorable à une instrumentalisation de sa diaspora dans le but d'obtenir des moyens financiers ou un soutien diplomatique, mais il est plus rare qu'il accueille avec bienveillance l'ingérence de celle-ci dans la gestion des affaires intérieures. Dans le cas kazakh, ce levier est d'abord économique. Les représentants de la diaspora en Turquie, aux États-Unis et en Europe occidentale possèdent une riche expérience de l'entreprise dans le monde capitaliste. Le succès des Kazakhs en Turquie est à cet égard un bon exemple puisque la communauté réunie en corporation a permis le développement de toute la filière du cuir en Turquie, en Grande-Bretagne, en France et dans d'autres pays. Forte de ces savoir-faire, elle est disposée à organiser des filières de production au Kazakhstan.

2 Durant la Première Guerre mondiale, par exemple, l'État allemand fit appel à sa diaspora aux États-Unis pour militer contre l'entrée en guerre des Américains aux côtés de la Triple Entente. 
Le troisième levier, d'après Esman (1990), réside dans la défense des droits et intérêts de la diaspora par le pays d'origine, selon le droit international. Il concerne essentiellement la migration ouvrière. De nombreux accords et traités interétatiques concernent les conditions de travail, confirmant le droit des gouvernements à protéger leurs compatriotes. C'est le cas du rôle qu'a joué l'État dans le rapatriement des Kazakhs d'Iran et d'Afghanistan qui ont commencé à revenir dans leur pays après les accords de février 1993 entre l'Iran et le Kazakhstan.

Il existe aussi un quatrième levier qui concerne le cas de deux groupes diasporiques respectivement installés dans le pays d'origine de l'un et de l'autre. On donnera pour exemple les Allemands de la Volga installés au Kazakhstan et les Kazakhs résidant en Allemagne. La situation de ces diasporas ethniques ne va pas sans jouer un rôle sur les relations entre les deux États. Ainsi, l'Allemagne qui accueille un petit nombre de Kazakhs, 178 familles tout au plus (soit près de 900 individus), les considère avec plus de tolérance et de bienveillance qu'elle ne le fait pour les immigrés turcs. Cette attitude ne s'explique pas seulement par la faiblesse numérique des Kazakhs d'Allemagne, mais aussi par la présence de centaines de milliers d'Allemands au Kazakhstan déportés dans les années 1940 après la fin de l'autonomie des Allemands de la Volga. Et si beaucoup d'entre eux sont retournés en Allemagne, à leurs yeux, le Kazakhstan demeure leur seconde patrie. Tous ces épisodes de l'histoire du peuple allemand pèsent dans la relation qu'il a avec la diaspora kazakhe en Allemagne. C'est la raison pour laquelle ces processus d'influence réciproque, tant sur le pays d'accueil que sur le pays d'origine, sont importants pour comprendre un des aspects des politiques internationales.

En 1992, devant le premier Congrès Mondial des Kazakhs, les médias ont commencé à parler de « diaspora » pour désigner l'ensemble des Kazakhs vivant au-delà des frontières du Kazakhstan. Or, en analysant les raisons qui font que $35 \%$ de nos compatriotes (soient 4,5 millions de Kazakhs) se retrouvent en dehors des frontières de la république, on s'aperçoit que le terme de « diaspora » ne peut s'appliquer à tous les groupes dispersés dans près de quarante pays. En effet, seulement 800000 d'entre eux, soit $18 \%$ des « co-ethniques », forment la diaspora, selon le sens qu'en donne Sheffer. 3,7 millions de Kazakhs de l'étranger vivent sur des territoires limitrophes du Kazakhstan : Russie (régions d'Astrakhan, Orenbourg, Kourgan, Omsk et République autonome de l'Altaï), Ouzbékistan (districts du Syr-Daria, Tchirtchik, Kyzylkoum, Myrzashol), Chine (districts de l'Altaï, Tarbagataï, l'Ili, Kouldja, Erenkabyrg, Barköl-Kumul et Province Autonome du Xinjiang-Ouïghour), ainsi que dans l'aïmak ou province de Bayan-Ölgiy en Mongolie. Ceux-là ne peuvent être assimilés à la « diaspora », puisqu'ils vivent là où ils ont toujours vécu. Mais la politique coloniale de l'empire tsariste aux XIXe et XXe siècles, et ensuite le régime totalitaire soviétique au XXe siècle, ont fait qu'ils se retrouvent aujourd'hui en dehors des frontières de leur pays. Ce sont eux que nous nommons irredenta (Mendikulova, 1995, 1996, 1997, 2006).

À la différence des diasporas, l'irredenta est un groupe qui, à un moment donné, s'est trouvé séparé de sa patrie par des guerres, des annexions, des contestations de frontières, des découpages coloniaux (Esman, 1990 : 83-84). Cette catégorie de la population était déjà connue des historiens du siècle passé qui la définissaient comme « le produit de la doctrine proclamant le droit de chaque peuple à s'instituer comme "nation" 》(Cobban, 
1969). C'est pourquoi j'ai proposé dans le cadre conceptuel des sciences sociales kazakhstanaises, le terme d'irredenta appliqué aux Kazakhs vivant sur des territoires limitrophes du Kazakhstan ${ }^{3}$.

L'irredenta kazakhe en Russie est le résultat de siècles de conquêtes territoriales et d'annexions depuis le XVIe siècle de territoires traditionnellement kazakhs - comme la conquête du khanat de Sibérie par Ermak -, ainsi que du découpage en 1924 de territoires du nord, de l'ouest et de l'est du Kazakhstan au profit de la RSFSR au moment de la délimitation des États-nations soviétiques d'Asie centrale (Mendikulova, 1995).

Les groupes kazakhs de Chine résultent quant à eux du conflit kazakho-djoungare entre les XVIe et XXe siècles ainsi que de l'expansion territoriale de l'empire russe qui ont mené à la redéfinition de la frontière sino-russe en Asie centrale. En conséquence, les Kazakhs ont perdu une partie importante de leur territoire au nord-est, à l'est, au sud-est et au sud du pays. Les populations de ces régions forment aujourd'hui l'irredenta kazakhe de Chine (Mendikulova, 2006).

Les Kazakhs d'Ouzbékistan sont le fruit de la refonte des frontières et des États en Asie centrale par le pouvoir russe en 1924 et du découpage de terres kazakhes au profit de l'Ouzbékistan dans les années 1950-1960. En effet, en 1924, lors de la formation stato-territoriale de l'ASSR du Kazakhstan et de ses voisins centrasiatiques, des populations kazakhes ayant toujours vécu dans les zones de Syr-Daria, Tachkent, Tchirtchik, Kyzylkoum et Myrzachol furent intégrées à l'Ouzbékistan. La région autonome du Karakalpakstan fut créée en 1925 et intégrée au Kazakhstan, mais en 1932, la modification territoriale et nationale de l'ASSR kazakhe entraîna la disparition de cette région au profit d'une nouvelle république autonome soviétique socialiste (ASSR) intégrée en 1936 à la RSS d'Ouzbékistan (Koblandin et Mendikulova, 2009).

\section{SPÉCIFICITÉS DE LA DIASPORA KAZAKHE}

Depuis les années 1960, les Kazakhs qui vivent à l'Ouest illustrent parfaitement le cas de la migration volontaire. Nous abordons ici les processus actuels auxquels la diaspora doit faire face : assimilation, acculturation, conservation de l'identité ethnique ou rapatriement. Deux tendances peuvent être observées : l'une relève de tentatives de conserver et de développer une identité ethnique tout en vivant en milieu étranger et l'autre dessine un net souhait de rapatriement au Kazakhstan, surtout depuis l'accès du pays à l'indépendance en 1991.

Une des caractéristiques de la communauté kazakhe à l'étranger, au moins jusqu'en 1991, réside dans l'absence de toute organisation communautaire structurée. Ceci peut s'expliquer par la faiblesse numérique des Kazakhs, mais aussi par leur engagement au sein d'organisations et d'associations turques. Ils n'ont alors pas ressenti le besoin de créer des structures propres permettant d'améliorer leur situation socio-économique,

3 Symposium international La Diaspora kazakhe : problèmes et perspectives (16-17 novembre 1995, à Türkistan), où je présentais une communication intitulée « Problèmes historiques de l'étude de la diaspora kazakhe » (Mendikulova, 1996 : 75-78). 
leur statut juridique et politique et de transmettre à la jeune génération les traditions et la culture kazakhes.

La situation évolua après la proclamation de l'indépendance du Kazakhstan qui provoqua chez tous les Kazakhs de l'étranger un sentiment de fierté d'appartenir à l'ethnie kazakhe. Depuis les années 1990 et l'apparition sur la mappemonde du Kazakhstan indépendant, le développement du pays est rapide et permet dans un futur proche d'espérer une entrée dans le club des cinquante premiers pays les plus développés. Cet événement a été le point de départ de la création d'associations dans divers pays ; ces dernières tentant de se mobiliser et de s'organiser pour mettre en œuvre des manifestations culturelles et sportives dans le but de contribuer à préserver l'identité ethnique. Ainsi dès l'été 1994, une société sportive basée à Munich organisa un championnat de football opposant cinq équipes de la diaspora : Berlin, Cologne et Munich, Paris et Vienne (Avrupa Kazak Türkleri Bülteni, 1994).

La diaspora n'est homogène, ni en termes sociaux, ni en termes économiques, culturels, juridiques ou éducatifs. Le plus souvent, elle se compose de Kazakhs originaires des trois pays d'origine où ils vivent traditionnellement : Kazakhstan, Russie, Chine ou pays d'immigration comme la Turquie. La langue étant l'un des marqueurs essentiels de l'ethnos, au cours de mes recherches sur l'identité linguistique, je suis arrivée à la conclusion que les Kazakhs de l'étranger se caractérisent par un trilinguisme voire un multilinguisme : outre le kazakh et la langue du pays d'accueil, les Kazakhs du Kazakhstan privilégient le russe, ceux du Xinjiang, le mandarin et le mongol et ceux de Turquie, le turc.

L'usage de la langue kazakhe par les deuxième et troisième générations de la diaspora vivant en Europe, aux États-Unis ou au Canada a un avenir incertain. La première génération (qui ne peut être qualifiée d' «âgée », puisque la moyenne d'âge oscille entre cinquante et cinquante-cinq ans et que ceux qui ont plus de soixante ans sont rares) connaît encore le kazakh mais déjà la génération suivante préfère utiliser au quotidien la langue du pays d'accueil. Bien que quelques jeunes aspirent à apprendre le kazakh, stimulés par le développement du pays, la langue d'origine est cependant moins usitée que d'autres langues. Inexistantes en France, aux États-Unis ou ailleurs, les écoles de langue sont le fait d'associations basées en Turquie ou en Grande-Bretagne. En outre, la question linguistique est étroitement liée au problème des trois alphabets utilisés par les Kazakhs des différents pays pour écrire leur langue. Les Kazakhs du Kazakhstan utilisent l'alphabet cyrillique, ceux de Turquie l'alphabet latin et ceux de Chine l'écriture arabe, ce qui complique considérablement la compréhension, l'approche des littératures et documents écrits, envoyés du Kazakhstan à destination des communautés kazakhes d'Europe occidentale.

En Amérique du Nord, les Kazakhs vivent majoritairement dans des familles nucléaires, car les enfants et l'épouse ont émigré dans le cadre du regroupement familial. En Turquie et dans d'autres pays du Moyen-Orient, ils préfèrent vivre dans des familles élargies : les parents vivent avec leurs enfants non mariés, mais aussi avec les fils mariés, les belles-filles et les petits-enfants. Cette situation est aussi peut-être la conséquence de la cherté du logement et de la terre, comme, par exemple, en Turquie, où il est plus économe et plus confortable de vivre ensemble ou quand les moyens manquent pour permettre au fils marié d'acquérir un logement. 
Les mariages intra-ethniques dominent dans les première et deuxième générations. Lors de nos entretiens dans différents pays, nous avons pu constater que la majorité n'accueille pas favorablement le mariage mixte. Le mariage entre turcophones est plus ou moins bien toléré, ce qui s'explique par des racines communes et laisse par conséquent présager d'une meilleure compatibilité et solidité du couple dans le mariage. Cette différenciation ethnique à propos des relations matrimoniales et familiales résulte d'une conscience de soi tendant à isoler les Kazakhs des autres ethnies quand ils sont en milieu étranger, tout autant qu'elle souligne l'appartenance à l'ethnos kazakh. En outre, les divisions tribales ont une signification particulière pour la préservation de la conscience ethnique et l'appartenance lignagère garde toute son importance en émigration. Le refus de mariages mixtes s'explique également par le non moins important facteur religieux, surtout pour les Kazakhs du Xinjiang, où l'Islam joue un rôle majeur. La religion musulmane recommande que chaque fidèle se marie avec l'un(e) de ses coreligionnaires : à défaut d'épouser un(e) musulman(e), il/elle préférera un(e) fidèle du Livre sacré, chrétien ou juif. Cependant, l'Islam recommande que les enfants nés de tels mariages, dans la mesure du possible, soient éduqués dans la foi musulmane.

Les membres des seconde et troisième générations qui ont passé leur jeunesse dans l'Europe occidentale des années 1990 ont un tout autre point de vue sur la question. Pour eux, la motivation ethno-religieuse n'est pas si importante. La tradition des mariages intra-ethniques (entre Kazakhs) laisse progressivement la place à des mariages interethniques et ce, pour différentes raisons : l'obtention de la nationalité du pays d'accueil, ou le choix de l'amour plutôt que celui de la raison. Le nombre des mariages mixtes est particulièrement important parmi les Kazakhs de France, mais, d'après le témoignage d'un représentant local de la diaspora, beaucoup de Kazakhs (venus de Turquie pour la plupart) se marient avec des Françaises, certains dans le but d'obtenir le statut de résident permanent. L'analyse des mariages interethniques chez les Kazakhs de la diaspora montre qu'avant tout ils concernent les hommes, arrivés seuls, sans famille, en Europe occidentale pour des raisons professionnelles ou économiques, pour des études ou un travail. La part des mariages mixtes impliquant une jeune fille augmente progressivement, mais il s'agit pour la plupart de jeunes filles du Kazakhstan et non de la diaspora, ce qui montre encore la réticence des vieilles générations à permettre de telles unions, même s’ils commencent à tolérer celles de jeunes hommes.

La diaspora kazakhe tente de préserver son identité ethnique, grâce aux liens étroits qu'elle entretient avec la mère-patrie historique et nombreux sont ses membres à vouloir visiter et connaître le pays pour y retrouver des parents et leurs racines. On observe aussi une tendance nouvelle au « retour » définitif au Kazakhstan.

\section{LA POLITIQUE DE LA RÉPUBLIQUE DU KAZAKHSTAN VIS-À-VIS DE LA DIASPORA}

Ce n'est qu'à l'indépendance du pays qu'il fut donné à la société kazakhstanaise d'étudier l'histoire et la situation actuelle des Kazakhs vivant dans les différents pays étrangers et d'établir des liens étroits avec eux. La politique de l'État vis-à-vis de la diaspora est liée au développement d'un Kazakhstan multiethnique, attentif non seulement 
à la consolidation et à l'unité du peuple kazakhstanais, comme communauté nouvelle d'individus vivant dans les frontières de la République, mais aussi à la défense de compatriotes que le destin a placés au-delà des frontières de la patrie historique.

À cet égard, l'Association Mondiale des Kazakhs joue un rôle majeur pour les communautés de l'étranger et se trouve au centre de la politique gouvernementale envers la diaspora et les irredenta. Après la proclamation de l'indépendance, il fut décidé de réunir au Kazakhstan les représentants de ces populations pour coordonner les actions de l'État en leur faveur. Le 29 septembre 1992 à Almaty, dans les salons du théâtre Abaï eut lieu le premier Congrès Mondial des Kazakhs auquel participèrent les représentants des Kazakhs vivant en Turquie, Allemagne, France, Suède, Norvège, Mongolie, Russie, Kirghizstan, Ouzbékistan et Turkménistan. Résolution fut prise d'œuvrer pour intensifier les relations entre le Kazakhstan et les Kazakhs de l'étranger et ce, dans tous les domaines. Seuls manquaient les Kazakhs de Chine que les autorités de Pékin n'avaient pas autorisés à se rendre à la manifestation. Ce jour-là, le gouvernement se posa en protecteur de tous les Kazakhs de par le monde. Cette initiative permit pour la première fois aux représentants de la diaspora et de l'irredenta, qui n'avaient pas eu l'occasion de fouler la terre de leurs ancêtres depuis des décennies, de se rencontrer et de connaître leurs compatriotes.

Le congrès entérina la création d'une nouvelle organisation pour coordonner les actions de toutes les communautés kazakhes expatriées, mais aussi pour étudier les spécificités historiques, ethnographiques, culturelles et linguistiques de la diaspora. C'est pourquoi, le 17 et 18 mars 1993, lors de la cinquième conférence de la société Kazakhstan fondée en 1976, on reconnut l'importance de donner aux Kazakhs de l'étranger l'opportunité de se familiariser avec leur histoire, leur culture d'origine et les avancées scientifiques et économiques du pays. Cette société devait ainsi apporter son aide aux expatriés pour soutenir les relations culturelles avec le pays. Le président de la République du Kazakhstan, Nursultan A. Nazarbaev fut élu président du bureau de l'Association Mondiale des Kazakhs et Kaldarbek Naïmanbaev en fut élu secrétaire général. La société Kazakhstan fut intégrée par décret à la nouvelle structure. Devenue le successeur légal de Kazakhstan, 1'Association hérita alors de son patrimoine et de sa documentation.

Le 14 février 1994 s'ouvrit à Almaty un centre d'affaires rattaché à l'Association et financé par des hommes d'affaires kazakhs de Turquie, de Russie et d'ailleurs, dans un objectif de développement commun. Elle prévoyait aussi l'ouverture d'un centre de presse et d'un centre culturel. En outre, il fut prévu, lors d'une session tenue à Almaty en mars 1994, d'attribuer, sur critères d'excellence, une vingtaine de bourses à des étudiants venant étudier au Kazakhstan.

À l'été 1994, l'Association conclut un accord avec des représentants du ministère de l'Instruction et des Établissements d'enseignement supérieur, invitant quarante Kazakhs de l'étranger à venir poursuivre leurs études au Kazakhstan. Grâce à des bourses, ces étudiants bénéficièrent d'une année d'études à Karatau (région de Djambul). L'Association obtint que le ministère aide ceux qui font le choix d'étudier au Kazalhstan ou le souhaitent. Dès l'année universitaire 1995-1996, 150 étudiants (dont des Kazakhs de Chine, Mongolie, Iran et Russie) bénéficièrent ainsi d'une aide pour suivre l'année préparatoire de l'Institut d'Agronomie d'Almaty, de l'Institut pédagogique d'État Abay, de l'Université 
d'État Abylay Khan ou des Départements de Langues des Facultés de Djambul, Türkistan et Taldykurgan (Deuletov, 2002 : 10). De 1995 à 1998, plus de 300 étudiants ont réussi l'année préparatoire et ont pu intégrer les facultés de leur spécialité. En octobre 1994, sous la direction du secrétaire général de l'Association, une délégation se rendit en Turquie, à Istanbul et Salihli, pour célébrer le grand poète kazakh Abaï, dont le Kazakhstan fêtait l'anniversaire sous l'égide de l'UNESCO. Les médias turcs ont souligné à l'époque l'importance de la manifestation pour la consolidation des relations entre les deux peuples. En mars 1995, à Istanbul se tint un Congrès des Kazakhs des pays de la région (Turquie, Kazakhstan, Ouzbékistan, Russie, Afghanistan et Iran) qui procéda au bilan de l'action de l'Association. Au cours des années 1990, plusieurs petits congrès préparatoires au Second Congrès Mondial des Kazakhs se tinrent en Russie, Chine, Mongolie et Ouzbékistan.

En 1995-1996, l'État adopta un programme d'aide à la diaspora. Ce programme visait le développement et la consolidation des relations de l'État avec la diaspora et l'irredenta. En janvier 1998, il fut question de créer un centre culturel de la diaspora en Europe, impliquant des représentants de tous les pays européens, dont la France. Ce centre, aujourd'hui basé à Cologne en Allemagne, compte quinze membres élus.

En 2001, l'ambassadeur de la République du Kazakhstan en France, en compagnie du maire de Nogent-sur-Marne, posait, le jour du dixième anniversaire de l'indépendance du Kazakhstan, une plaque commémorative devant la maison où vécut Mustafa Tchokaï. Le Premier ministre du Kazakhstan et des vétérans de la Seconde Guerre mondiale ainsi que des représentants de la diaspora kazakhe en France étaient présents à la cérémonie. Cette visite officielle fut également l'occasion d'obtenir pour les Archives Nationales du Kazakhstan des copies du Fonds Mustafa Tchokaï conservé à l'INALCO4 .

Le 20 juillet 2002, le Premier ministre signa un décret officialisant la future tenue du Second Congrès Mondial des Kazakhs qui se déroula les 23 et 24 octobre 2002 (Deouletov, 2003). D'après l'Association, il réunit plus de 400 participants venus de trente-quatre pays différents. La délégation la plus importante venait de l'Ouzbékistan voisin. Pour la première fois étaient présents des représentants des Kazakhs de Chine. Le président de la République, dans son discours inaugural, rappela les grandes orientations et priorités de la politique intérieure et extérieure du Kazakhstan et son aspiration à rencontrer les Kazakhs vivant à l'étranger lors de ses visites officielles. Le Congrès élabora un nouveau programme gouvernemental pour l'assistance aux expatriés, examinant également la question du rapatriement pour ceux qui souhaitaient revenir dans leur pays d'origine. À l'issue du Congrès, 800 familles kazakhes de Mongolie avaient déjà exprimé ce souhait. En 2004-2005, l’État dut établir un quota maximal de 15000 rapatriés, tant la demande était importante : près de 3 millions de Kazakhs expatriés disent envisager leur retour définitif.

Selon les données du Comité des Migrations auprès du ministère du Travail et de la Protection sociale, en dix ans, près de 600000 Kazakhs ethniques sont revenus au pays en bénéficiant du dispositif de retour par quota officiel, mais des sources officieuses

4 Mustafa Tchokaï (1890-1941), intellectuel turkestanais d'origine kazakhe, éphémère président des États autonomes de Kokand et d'Alash Orda, réfugié en France, a longtemps animé la résistance turkestanaise comme l'URSS. Il a vécu une grande part de sa vie à Nogent-sur-Marne [Ndlr]. 
avancent le chiffre de 1500000 rapatriés. Pour les seules années 1991-1992, où pourtant la situation économique du pays était critique, le nombre des oralman (en kazakh les personnes optant pour un retour volontaire ${ }^{5}$ ) dépassait les 60000 individus.

C'est le 18 mai 2005 que l'Association créa le Centre pour la Jeunesse étudiante, auprès de l'Association Mondiale des Kazakhs à la suite de rencontres entre spécialistes portant notamment sur la création d'un Centre de recherche consacré à l'étude des diasporas au sein de l'Association.

L'année 2005 a été marquée par la tenue à Astana, nouvelle capitale du pays, du troisième Congrès Mondial des Kazakhs organisé par un décret gouvernemental signé par le Premier ministre 6 . Il a réuni 300 délégués de trente-deux pays (Russie, Chine, Autriche, Norvège, Tchéquie, Italie, Singapour, Égypte, etc.), 200 représentants de toutes les provinces du pays et des villes d'Almaty et Astana, ainsi que les médias ${ }^{7}$. L'image retenue a été celle des délégués plantant des jeunes pousses d'arbres le long de l'avenue Atameken ${ }^{8}$. Lors de ce congrès, quatre ateliers, organisés par les représentants de différents ministères, ont été consacrés au développement de la diaspora et de la culture kazakhe, aux études supérieures pour les étudiants de la diaspora au Kazakhstan, au placement des investissements de la diaspora et à la politique migratoire sur la question des oralman. Des demandes d'enseignants spécialisés ont été formulées concernant les enseignements en langue kazakhe avec la mise à disposition de manuels et de matériel pédagogique. D'autres débats eurent lieu sur l'octroi de la citoyenneté kazakhstanaise aux oralman, sur la détermination de leur statut et sur l'assistance et les services aux rapatriés afin de leur garantir un logement ou un terrain ainsi qu'une insertion professionnelle. Les dernières questions abordées portaient sur les opportunités d'investissements au pays d'origine et sur les contributions des entrepreneurs kazakhs de l'étranger dans le développement des PME.

5 Voir la contribution de Gaëlle Lacaze dans ce même numéro.

6 Le vice-Premier ministre, A.S. Essimov, fut nommé président de la commission, avec comme adjoints : E.M. Kossoubaev, ministre de la Culture, de l'Information et des Sports, T.A. Mamashev, secrétaire général du Bureau de l'Association Mondiale des Kazakhs, O.E. Shoukeev, maire de la ville d'Astana, D.A. Kaletaev, chef du Département de Politique sociale au secrétariat de la présidence de la république. B.J. Omarov, président de la Commission pour les Langues, fut nommé secrétaire de la commission, montrant ainsi l'intérêt très direct du pouvoir central pour cette question.

7 Beaucoup de jeunes étaient présents, qu'ils soient hommes d'affaires, scientifiques, enseignants, médecins, étudiants, artistes, c'est-à-dire les personnes socialement, économiquement et culturellement mobiles parmi les actifs de ces communautés kazakhes de l'étranger. Parmi les délégués, on comptait vingt scientifiques, cinq membres du Parlement, un haut fonctionnaire, huit fonctionnaires de province, trente-neuf présidents de centres culturels kazakhs à l'étranger.

8 Avenue « de la Patrie » au centre de la nouvelle capitale, construite à partir de la ville provinciale d'Akmolinsk, rebaptisée Astana. Le nouvel état construit là une capitale moderne sur deux principes destinés à affermir son autorité : vitrine d'un Kazakhstan entreprenant, moderne, puissant, et recentrage vers le nord d'un territoire jusqu'alors déséquilibré ; le nord où se situe Astana étant bien plus russifié que le sud. De plus, Almaty est proche de la frontière kirghize et non loin de la Chine. Cette opération d'urbanisme volontaire peut être comparée au transfert de la capitale turque d'Istanbul à Ankara ou à la construction de Brasilia ou Canberra [Ndlr]. 
Lors de la séance inaugurale dans les nouveaux bâtiments de la Maison du Gouvernement, le président du Kazakhstan et le président du bureau de l'Association Mondiale des Kazakhs avaient mis l'accent sur le développement du pays et sur les communautés kazakhes de l'étranger considérés comme central dans la politique nationale. On peut donc noter une nette amélioration des relations et des échanges entre le pays et la diaspora ces dernières années, même si des problèmes subsistent quant à la garantie des intérêts de la diaspora liés au droit, à la culture, à l'information et enfin à l'éducation. La visite par les délégués de la nouvelle résidence du chef de l'État, $A k$ Orda ${ }^{9}$, motif de fierté pour les Kazakhs, a été un événement marquant, car les petits et de grands congrès mondiaux ne sont pas de simples festivités, mais d'importantes manifestations politiques pour la consolidation de la société kazakhstanaise, moments emblématiques de la construction de l'État-nation.

Le décret présidentiel de 2005 (Programme officiel d'assistance aux compatriotes, vivant à l'étranger pour 2005-2007) marque une étape importante dans le développement des relations entre l'État kazakh et la diaspora kazakhe. L'objectif de ce Programme est de "créer un système étatique de défense et de soutien juridique, économique et social aux compatriotes kazakhs, vivant à l'étranger, mais demeurant une partie indivisible de la nation kazakhe ». Pour y parvenir, le président de la République propose de mettre en œuvre plusieurs chantiers : le perfectionnement des normes juridiques concernant le soutien aux compatriotes, vivant à l'étranger ; l'établissement de liens économiques solides ; le soutien de relations culturelles et sociales de solidarité stables ; l'information des compatriotes ; la facilitation des études au sein d'établissements supérieurs au Kazakhstan ; 1'assistance à la consolidation de la diaspora kazakhe.

Pour 2011, l'Association Mondiale des Kazakhs a proposé une série de manifestations et d'actions à long terme consacrées à la consolidation de la diaspora et de l'irredenta kazakhes dans les domaines de la science, de l'éducation, de la culture, de la spiritualité, du développement économique et social et de l'essor des centres culturels. Ces actions visent toutes à préserver l'identité ethnique des Kazakhs de l'étranger et à favoriser l'étude de la langue maternelle, de l'histoire du Kazakhstan, des traditions et coutumes nationales.

9 Le nom du palais présidentiel, Ak Orda (Horde Blanche), est polysémique, il renvoie à la vie nomade des origines, aux états nomades impériaux dont le plus prestigieux fut celui de Gengis Khan. Se reporter aux références historiques médiévales comme la Horde d'Or, la Horde Bleue et aux dénominations - erronées d'ailleurs - comme celles des confédérations kazakhes d'avant l'arrivée des Russes dans la steppe : Grande, Petite et Moyenne Hordes. Si ce n'est que le kazakh emploie jüz (centurie) et non orda (armée, camp du khan, par extension état). Cf. le turc ordu (armée) [Ndlr]. 


\section{POLITIQUE DE RAPATRIEMENT DE LA RÉPUBLIQUE DU KAZAKHSTAN}

Le rapatriement des Kazakhs ethniques ${ }^{10}$ a commencé dès 1992. Deux vagues de retour peuvent être distinguées : la première va de 1992 à 1997, date de l'entrée en vigueur de la loi sur les migrations de 1997 et la seconde de 1997 à nos jours, date à laquelle les oralman peuvent se réinstaller au Kazakhstan (Mendikulova, 2004 : 3-35). Dans les années 1990, la majeure partie des rapatriés venait de Russie, de Mongolie, de Turquie, du Tadjikistan, d'Iran et d'Afghanistan (Diener, 2009). Depuis les années 2000, les retours concernent surtout des Kazakhs d'Ouzbékistan et du Turkménistan et depuis 2003, de Chine.

La diaspora kazakhe en Iran se compose de descendants d'émigrés ayant quitté le pays pendant la Guerre civile de 1918-1920 ou pendant la collectivisation de 19291932. Parmi eux figurent des Kazakhs d'Afghanistan, réfugiés en Iran depuis les années 1980. Ces derniers, considérés comme des réfugiés de guerre, ont vécu jusqu'en 1993 sans papiers et sans aucune assistance de la part du gouvernement iranien, raison pour laquelle le Kazakhstan négocia avec les autorités iraniennes leur rapatriement. En 1993, plusieurs milliers de Kazakhs d'Iran furent donc autorisés à se réinstaller au Kazakhstan. En 1996, une Loi sur l'Immigration instituait le statut de "réfugié » pour tout Kazakh ethnique rapatrié. Selon l'article 17, "le statut de réfugié est attribué à toute personne de la diaspora vivant à l'étranger ayant des liens avec la patrie historique, mais obligée de revenir au Kazakhstan car menacée d'oppression, de poursuites, de restrictions de ses droits et libertés ». Le gouvernement créa alors un département des Migrations auprès du ministère du Travail et de la Protection sociale chargé de l'organisation de ces retours. La Loi de 1997 sur les migrations stipule que chaque candidat au rapatriement reçoit le statut d'oralman. De 1993 à 2009, plus de 700000 personnes sont ainsi retournées au Kazakhstan (Statistical Yearbook of Kazakhstan, 2007 : 26-28 ; IA Novosti-Kazakhstan, 18/01/2010 ; Mendikulova, 2009). En 1998, le gouvernement adopta un Code de rapatriement des Kazakhs ethniques dans leur mère-patrie historique. L'objectif principal de ce Code est de mettre en place des procédures de rapatriement pour les Kazakhs ethniques en aidant à leur réinstallation et à leur intégration professionnelle et sociale.

Il existe au Kazakhstan une procédure d'acquisition du statut d'oralman. L'article 14 de la Loi sur La migration de la population stipule que «les personnes souhaitant obtenir ce statut doivent transmettre leur requête en mains propres à un membre majeur de leur famille, à un représentant compétent du corps diplomatique ou consulaire de la République du Kazakhstan à l'étranger ou encore à l'Agence des Migrations et de la Démographie ». La loi reconnaît comme « parent », les enfants (y compris adoptés), les époux ou les épouses, la fratrie, les ascendants et leur famille ainsi que la descendance

10 La politique de rapatriement des Kazakhs ethniques est régie par les Lois républicaines sur $L a$ citoyenneté dans la république du Kazakhstan, De l'immigration (26 juin 1992), De la migration de population (13 décembre 1997) et par le décret du cabinet des ministres De la procédure et conditions de réinstallation dans la RSS du Kazakhstan d'individus de nationalité kazakhe, qui avaient exprimé le souhait de travailler dans les zones rurales d'autres républiques et à l'étranger. 
ayant atteint la majorité. Chaque année, le quota d'immigration permet à 5000 familles, soit près de 15000 individus de bénéficier de ce statut. En 2010, le quota annuel a été revu à la hausse pour permettre l'accueil de 20000 nouveaux oralman.

\section{Nombre d'Oralman revenus au Kazakhstan, par régions (Période de 1991 au $1^{\text {er }}$ octobre 2008)}

\begin{tabular}{|c|l|c|c|c|c|c|c|}
\hline \multirow{2}{*}{$\mathrm{N}^{\circ}$} & \multirow{2}{*}{ Région } & \multicolumn{3}{|c|}{ Total } & \multicolumn{4}{c|}{ Répartition par sexe } \\
\cline { 2 - 8 } & Familles & Personnes & Hommes & $\%$ & Femmes & $\%$ \\
\hline 1 & Akmola & 7451 & 38233 & 17674 & 46,2 & 20559 & 53,8 \\
\hline 2 & Aktyube & 6819 & 33759 & 15584 & 46,2 & 18175 & 53,8 \\
\hline 3 & Almaty (Région) & 30049 & 102023 & 49749 & 48,8 & 52274 & 51,2 \\
\hline 4 & Atyrau & 3656 & 14998 & 7250 & 48,3 & 7748 & 51,7 \\
\hline 5 & $\begin{array}{l}\text { Kazakhstan } \\
\text { oriental }\end{array}$ & 8945 & 35069 & 15925 & 45,4 & 19144 & 54,6 \\
\hline 6 & Jambyl & 11123 & 63983 & 31351 & 49,0 & 32632 & 51,0 \\
\hline 7 & $\begin{array}{l}\text { Kazakhstan } \\
\text { occidental }\end{array}$ & 3172 & 14234 & 6902 & 48,5 & 7332 & 51,5 \\
\hline 8 & Karaganda & 12998 & 46273 & 22311 & 48,2 & 23962 & 51,8 \\
\hline 9 & Kostanai & 5891 & 24573 & 11795 & 48,0 & 12778 & 52,0 \\
\hline 10 & Kyzylorda & 3542 & 17314 & 7943 & 45,9 & 9371 & 54,1 \\
\hline 11 & Mangystau & 24460 & 85464 & 34727 & 40,6 & 50737 & 59,4 \\
\hline 12 & Pavlodar & 6175 & 28072 & 13064 & 46,5 & 15008 & 53,5 \\
\hline 13 & $\begin{array}{l}\text { Kazakhstan } \\
\text { septentrional }\end{array}$ & 6439 & 33951 & 14752 & 43,5 & 19199 & 56,5 \\
\hline 14 & $\begin{array}{l}\text { Kazakhstan } \\
\text { méridional }\end{array}$ & 45827 & 155963 & 73438 & 47,1 & 82525 & 52,9 \\
\hline 15 & Ville d'Almaty & 1778 & 6471 & 3162 & 48,9 & 3309 & 51,1 \\
\hline 16 & Ville d'Astana & 1199 & 5661 & 2632 & 46,5 & 3029 & 53,5 \\
\hline & \multicolumn{1}{|c|}{ Total } & 179524 & 706041 & 328259 & 46,5 & 377782 & 53,5 \\
\hline
\end{tabular}

Source : Agence des Statistiques de la République du Kazakhstan, Données : 1991 - $1^{\text {er }}$ octobre 2008 ; Kazak Eli (2009), $24^{\text {th }}$ Nauryz, 5-6.

En outre, des oralman reviennent par leurs propres moyens au Kazakhstan, et ce sont eux qui rencontrent les plus grandes difficultés pour s'installer dans le pays. Mais, 
qu'ils entrent dans le cadre des quotas d'immigration ou qu'ils reviennent spontanément, l'État garantit à tous des conditions avantageuses de réinstallation : aides dans la recherche d'un emploi et d'un logement, attributions de pensions et d'allocations conformes à la législation sociale, bourses pour des études supérieures, offres de terrains constructibles ou agricoles. Les services de l'État organisent aussi des séminaires de formation sur les aspects juridiques et culturels.

\section{CONCLUSION}

La politique officielle de la république kazakhe vis-à-vis de la diaspora ou du rapatriement des oralman a certes évolué, mais elle demeure inchangée dans ses objectifs principaux. Elle œuvre pour l'amélioration des conditions d'accueil et de vie des oralman et celle des Kazakhs vivant au-delà de ses frontières. Certes des lacunes subsistent dans cette prise en charge par les autorités locales notamment, mais cette politique va en sens contraire de ceux qui ont tendance à critiquer les priorités de la politique de l'État kazakh concernant la diaspora, l'irredenta et les rapatriés.

\section{Références bibliographiques}

ABADAN-UNAT Nermin (1985) Identity Crisis of Turkish Migrants. First and Second Generation, in İlhan Başgöz and Norman Furniss Dir., Turkish Workers in Europe. An Interdisciplinary Study, Indiana University Turkish Studies, 5, Bloomington Indiana, pp. 3-22.

Agence des Statistiques de la République du Kazakhstan (2005), Rapport démographique annuel du Kazakhstan, 2004. Rapports annuels, Almaty, Agence des Statistiques de la République du Kazakhstan, 448 p. (en russe).

Agence des Statistiques de la République du Kazakhstan (2007) Rapport statistique du Kazakhstan. 2007, Astana, Agence des Statistiques de la République du Kazakhstan, 494 p. (en russe).

ASHKENASI A. (1990) Nationalism and National Identity, B.; Das arab. Buch, Ethnizitat und Ges, Occasional papers, 23, $250 \mathrm{p}$.

Avrupa Kazak Türkleri Bülteni (1994), Munich, 2.

CASTLES S. and KOSACK G. (1973) Immigrant Workers and Class Structure in Western Europe, London, $268 \mathrm{p}$.

COBBAN Alfred (1969) The Nation State and National Self Determination, New York, 189 p.

Diasporas in International Politics (1986) London, Groom-Helm Publishers, 189 p.

DIENER Alexander (2009) One Home or Two? The Nationalization and Transnationalization of Mongolia's Kazakhs, Washington DC, Woodrow Wilson Center Press, Stanford, Stanford University Press, $405 \mathrm{p}$.

ДӘУЛЕТОВ Т. (2002) Дүниежүзі қ̧азақтары қ̧ауымдастывыңын шежіресі, Алматы, ДҚҚ, 1046.

ДӘУЛЕТОВ Т. (2005) Дүниежүзі ққазақтары қ̧ауымдастывыңын шежіресі (29/07/2002 29/09/2005). II kiman, Алматы, ДҚҚ, 115 б.

ДӘУЛЕТОВ Т. (2003) Дүниежүзі қ̧азақтарының II құрылтайы, Алматы, 77 б.

ENGELBREKTSSON U.-B. (1978) The Force of Tradition. Turkish Migrants at Home and Abroad, Göteborg, 309 p.

ESMAN Milton J. (1990) Ethnic Pluralism and International Relations, Canadian Review of Studies in Nationalism, XVII (1-2), pp. 83-88. 
IA Novosti-Kazakhstan (2010), 18 January.

ҚАЗАҚ ЕЛІ (1996) Мамырдың 13-і, с. 2.

ҚАЗАҚ ЕЛІ (1997) Мамырдың 30-ы, с. 5.

KAZAKH ELI (2009) $24{ }^{\text {th }}$ Nauryz, 5-6.

ҚОБЛАНДИН Қ.И., МЕНДІҚҰЛОВА Г.М. (2009) Өзбекстандагы қ̧азақтардың тарихы және бүгінгі дамуы, Алматы, ДҚҚ, 294 б.

КСЁНДЗЫК Н.Н. (1991) Туреикая трудовая иммиграџия в странах Западной Европь (70-80 г2.), Киев, Наукова думка, 108 с.

Law of the Republic of Kazakhstan about Migration (1998) Zakon Respubliki Kazakhstan ot 13 Dekabrya 1997 goda № 204-I. O Migratsii naseleniya, Almaty, 121 p.

Loi de la République du Kazakhstan sur les migrations, du 13 décembre 1997, n²04-I. De la migration de la population (1998) Almaty, $121 \mathrm{p}$ (en russe).

MENDIKULOVA Gulnara (2009) Contemporary Migration Trends between Kazakhstan and China, Proceeding of International Conference "Central Asia-China: Conditions and Perspectives of Cooperation”, 4-5 th June 2008, Almaty, KISI, pp. 158-170.

МЕНДИКУЛОВА Г.М. (2006) Казахская диаспора: история и современность, Алматы, Реиз, $343 \mathrm{c}$.

MENDIKULOVA Gulnara (2000) Repatriation into Kazakhstan: History and Current Situation. International Conference on Central Asia, Toronto, 6, pp. 77-81.

MENDIKULOVA Gulnara (2005) Repatriation of the Kazakhs from Turkey into Sovereign Kazakhstan, Interrelations of Turkey and Central Asia in the Context of Enlarging Europe, Almaty, pp. 257-266 (in Russian).

MENDIKULOVA Gulnara (2004) Some Problems of Adaptation of the Kazakh Repatriates (Oralmans) in Contemporary Kazakhstan, Otan tarikhy, 2, pp. 3-35.

МЕНДИКУЛОВА Г.М. (2000а) Актуальные проблемы диаспорологии в Казахстане, Отан тарихы, 3-4, с. 37-42.

МЕНДИКУЛОВА Г.М. (1997) Исторические судьбы казахской диаспоры. Происхождение и развитие, Алматы, Гылым, 262 с.

МЕНДИКУЛОВА Г.М. (1998) Казахская диаспора и Республика Казахстан: проблемы и перспективы, Казахстан-Спектр, 1, с. 73-77.

МЕНДИКУЛОВА Г.М. (1995) Казахская ирредента в России (история и современность), Евразийское сообщество, 8, с. 70-80.

МЕНДИКУЛОВА Г.М. (1996) Международный симпозиум “Казахская диаспора: проблемы и перспективы”, Вестник Министерства науки-Академии наук Республики Казахстан, 2, c. 75-78.

SHEFFER Gabriel (Ed.) (1986) Modern Diasporas in International Politics, London \& Sydney, Palgrave-Macmillan, $349 \mathrm{p}$.

TAPIA Stéphane de, AKGÖNÜL Samim (Dir.) (2007) Kazakhs, Kalmouks et Tibétains en France, Paris, L'Harmattan (Compétences interculturelles), 273 p.

ФРОЛКИН Н.М. (1975) Трудовая иммиграция во Франции в новейшее время, Киев, Наукова думка, 285 с. 